\title{
Progresión del deterioro cognitivo y su relación con factores de riesgo modificables en una cohorte de adultos
}

\author{
Progression of cognitive impairment and its \\ relation to modifiable risk factors \\ in a cohort of adults
}

Olga lucía Pedraza-Linares, María Isabel Cedeño-Izquierdo, Lyda Carolina Sarmiento-Borda, luz Adriana Santamaría-Ávila, José Javier González-Arteaga, Ana María Salazar-Montes, María Camila Montalvo-Villegas, Ángela Gisselle Lozano-Ruiz, Isis Norella Camacho-Bermúdez, Pablo César Castillo- Homez, Katherine Grimaldo-Lizarazo, César Arcadio Piñeros-Perilla, Fabio Alexánder Sierra-Matamoros • Bogotá, D.C. (Colombia).

\section{Resumen}

Un estudio de corte transversal realizado entre 2012-2014 en adultos autónomos de Bogotá, mostró una prevalencia de $23 \%$ de demencia, encontrándose asociada con baja escolaridad, edad avanzada e hipertensión arterial. La relación de estos factores de riesgo con la progresión del deterioro cognitivo no ha sido estudiada en nuestra población.

Objetivo: evaluar la asociación entre factores de riesgo cardiometabólicos o sociales con la progresión a deterioro cognitivo leve (DCL) o demencia, en adultos autónomos de Bogotá.

Material y métodos: una cohorte de sujetos normales y con DCL, del estudio de 2012-2014, se revaluó aplicándose el protocolo neuropsiquiátrico y neuropsicológico del estudio anterior. Se realizaron análisis de correspondencia múltiple y de regresión logística.

Resultados: se revaluaron 215 adultos autónomos, 118 sujetos habían sido diagnosticados con cognición normal y 97 con DCL en el primer estudio; $73 \%$ fueron mujeres con edad promedio de 71(DE:7.3) años y escolaridad de 8.2 (DE: 5.4) años. Progresaron a demencia $6 \%$ de los sujetos en un tiempo de 4(DE:1) años. Un $75 \%$ de sujetos normales permaneció sin cambio y $22 \%$ progresó a DCL; mientras que $65 \%$ de sujetos con DCL no tuvo cambios y un $25 \%$ se normalizó. Progresar de normal a DCL se asoció con baja escolaridad OR=2.43 (IC95\% 1.004-5.91; p=0.049) y de DCL a demencia con $\mathrm{IMC} \leq 25 \mathrm{OR}=6.3$ (IC95\% 1.26-31; $\mathrm{p}=0.025)$.

Conclusión: tener baja escolaridad ( $\leq 5$ años) se asoció en los sujetos normales con un mayor riesgo de progresión a DCL, mientras que tener un IMC $\leq 25$ aumentó el riesgo de progresión a demencia en los sujetos con DCL. No identificamos factores protectores en los sujetos que se normalizaron. (Acta Med Colomb 2019; 44: 66-74).

Palabras clave: progresión o recuperación, deterioro cognitivo leve, demencia, factores de riesgo cardiometabólicos, factores de riesgo sociales.

\footnotetext{
Abstract

A cross-sectional study carried out between 2012-2014 in autonomous adults of Bogotá showed a $23 \%$ prevalence of dementia, being associated with low schooling, advanced age and arterial hypertension. The relationship between these risk factors and the progression of cognitive deterioration has not been studied in our population.

Objective: to evaluate the association between cardio-metabolic or social risk factors with the progression to mild cognitive impairment (MCI) or dementia, in autonomous adults of Bogotá.

Material and methods: a cohort of normal subjects with MCI, from the 2012-2014 study was re-evaluated applying the neuropsychiatric and neuropsychological protocol of the previous study. Multiple correspondence and logistic regression analyzes were performed.
}

Dra. Olga Lucía Pedraza-Linares: Profesora Titular, Grupo de Neurociencias, Grupo Interdisciplinario de Memoria; Dras. María Isabel Cedeño-Izquierdo y Lyda Carolina SarmientoBorda: Residentes Tercer Año de Medicina Familiar; Dres.: Luz Adriana Santamaría-Ávila y José Javier González-Arteaga: Especialistas en Medicina Familiar; Ana María SalazarMontes, María Camila Montalvo-Villegas, Ángela Gisselle Lozano-Ruiz, Isis Norella Camacho-Bermúdez, Pablo César CastilloHomez, Katherine Grimaldo-Lizarazo: Grupo de Neurociencias, Grupo Interdisciplinario de Memoria; Dr. César Arcadio Piñeros-Perilla: Instructor Asociado; Dr. Fabio Alexánder Sierra-Matamoros: Instructor Asistente. Fundación Universitaria de Ciencias de la Salud, Hospital Infantil Universitario de San José (HIUSJ). Bogotá, D.C. (Colombia). Correspondencia: Dra. Olga Lucía Pedraza. Bogotá, D.C. (Colombia).

E-mail: opedraza@hospitalinfantildesanjose. org.co

Recibido: 25/V/2018 Aceptado: 22/III/2019 
Results: 215 autonomous adults were re-evaluated; 118 subjects had been diagnosed with normal cognition and 97 with MCI in the first study; $73 \%$ were women with an average age of 71 (SD: 7.3 ) years and schooling of 8.2 (SD: 5.4 ) years. $6 \%$ of the subjects progressed to dementia in a time of 4 (SD: 1) years. $75 \%$ of normal subjects remained unchanged and $22 \%$ progressed to MCI while $65 \%$ of subjects with MCI did not change and $25 \%$ normalized. Progressing from normal to MCI was associated with low schooling OR $=2.43$ (95\% CI 1.004-5.91; $\mathrm{p}=0.049)$ and from MCI to dementia with $\mathrm{BMI} \leq 25 \mathrm{OR}=6.3$ (IC95\% 1.26-31; $\mathrm{p}=0.025)$.

Conclusion: having low schooling ( $\leq 5$ years) was associated in normal subjects with a higher risk of progression to $\mathrm{MCI}$, while having a $\mathrm{BMI} \leq 25$ increased the risk of progression to dementia in subjects with MCI. Protective factors in subjects who normalized were not identified. (Acta Med Colomb 2019; 44: 66-74).

Keywords: progression or recovery, mild cognitive impairment, dementia, cardio-metabolic risk factors, social risk factors.

\section{Introducción}

El alto índice de envejecimiento de la población mundial se acompaña de una progresión significativa de la tasa de demencia (1). El estudio Global Burden of Disease 2010, estimó la demencia como la tercera causa de años vividos con discapacidad a nivel mundial (2). La demencia representa una carga importante para los sistemas de salud, debido a las complicaciones y el alto grado de discapacidad que puede llegar a generar (3).

En 2015, se estimó la cantidad de personas con demencia en cerca de 46.8 millones a nivel mundial, calculándose que para el 2050 lleguen a ser de 131.8 millones, lo que generará un grave problema de salud pública (4). Alzheimer's Disease International informó la prevalencia de demencia en el Norte de África y Medio Oriente como la más alta a nivel mundial, siendo de $8.7 \%$ en personas mayores de 60 años; le seguirían los países latinoamericanos con $8.4 \%$ (5). Sin embargo, existen estudios que sugieren prevalencias que irían desde $17.5 \%$ hasta $26.4 \%(6-8)$.

El bajo nivel educativo ( $\leq 5$ años) se ha considerado como uno de los factores de riesgo más importante en diferentes estudios epidemiológicos sobre demencia; y este riesgo de demencia disminuiría en un $7 \%$ por cada año de educación $(9,10)$.

En el estudio de César et al. 2016 los autores describen una tasa del analfabetismo entre ancianos latinoamericanos de $9.3 \%$, con una prevalencia de demencia dos veces mayor que en sujetos alfabetizados (6). Según el estudio SABE en Colombia, la población adulta mayor presentó un promedio de escolaridad de 5.5 años, y menos de $1 \%$ se encontraba estudiando. Niveles educativos más bajos se observaron a medida que aumentaba la edad, especialmente en la zona Atlántica (11).

El estudio realizado entre 2012 y 2014 por el grupo de Neurociencias de la Fundación Universitaria de Ciencias de la Salud (FUCS) en población adulta autónoma de Bogotá, encontró una prevalencia de $23 \%$ de demencia en mayores de 50 años, coincidiendo con lo descrito por Gooding y cols. 2006 , en su estudio realizado en el Huila $(12,13)$.
La alta prevalencia de demencia del grupo estudiado, se asoció especialmente con la baja escolaridad ( $\leq 5$ años), además de la edad y la hipertensión; esta prevalencia y la descrita por Gooding et al. 2006, contrastan con lo descrito en estudios previos realizados en Colombia (12-16).

Un estudio de cohorte de adultos mayores entre 70 y 90 años realizado en Sydney durante dos años muestra que el deterioro observado con el envejecimiento normal aumenta en el tiempo e involucra funciones intelectuales como la velocidad de procesamiento y transmisión de la información, la atención, la memoria y las funciones ejecutivas; así mismo el deterioro cognitivo leve y la demencia se asocian también con edades mayores, depresión y enfermedad vascular entre otros (17).

Por su parte, la hipertensión arterial diagnosticada entre los 65-70 años aumenta el riesgo de deterioro cognitivo. Sin embargo, en pacientes mayores de 75 años, tener una presión arterial sistólica (PAS) baja, se convierte en un factor de riesgo para demencia, mientras que la presión arterial diastólica (PAD) alta sería un factor protector; y un $77 \%$ de población adulta mayor con variabilidades en la PAS presentaría demencia con mayor frecuencia. Cuando la hipertensión se presenta en la edad media de la vida se asocia principalmente a demencia vascular (18).

En la población adulta bogotana estudiada entre 2012 y 2014 se encontró un OR de 1.45 (95 \% IC: 1.03-2.05) para demencia en personas hipertensas (12).

En cuanto a la diabetes mellitus se ha descrito que, en las edades media y tardía de la vida, se asocia con mayor riesgo de deterioro cognitivo y demencia, especialmente con demencia vascular (19).

En cuanto a los estudios que evalúan la relación entre obesidad y dislipidemia con deterioro cognitivo, los resultados aún son controversiales $(19,20)$. El estudio de Qizilbash et al. (2015) sugiere que un índice de masa corporal (IMC) alto en la edad media de la vida, aumentaría el riesgo de demencia, mientras que un IMC bajo (menor de 20) en la edad tardía, aumentaría dicho riesgo y un IMC alto sería protector en este mismo periodo $(21,22)$. El síndrome 
metabólico, que es una agrupación de factores de riesgo cardiovascular (obesidad, diabetes mellitus, hipertensión arterial y dislipidemia), también se ha descrito asociado con un mayor riesgo de deterioro cognitivo leve (DCL) y progresión a demencia (19).

Además de los factores cardiovasculares y metabólicos, se ha sugerido que factores sociales como el matrimonio funcionarían como un factor protector y reducirían el riesgo de DCL o demencia, mientras que una baja funcionalidad social (entendida como escasa actividad social, vivir solo, o tener una red social de apoyo deficiente) sería por su parte, un factor de riesgo para el deterioro cognitivo $(17,23)$.

El objetivo del presente estudio fue evaluar si existe asociación entre los cambios cognitivos observados en el tiempo, en sujetos que habían sido encontrados normales o con DCL en el estudio realizado entre 2012 y 2014, con sus factores de riesgos cardiometabólicos o sociales.

\section{Material y métodos}

Diseño: el presente es un estudio de cohorte en el que se incluyeron pacientes del proyecto "Prevalencia y caracterización del DCL y Demencia en una muestra de adultos mayores de 50 años de Bogotá 2012- 2014" realizado por el grupo de Neurociencias de la FUCS $(12,16)$, en quienes se evaluó como desenlace el cambio en su estado cognitivo después de cuatro años de seguimiento y se analizó la relación entre este desenlace y factores de riesgo cardiovascular y metabólicos.

\section{Participantes}

Del estudio anterior se dispone de una población de 423 adultos que fueron evaluados en segunda fase con el protocolo completo de la clínica de memoria, encontrándose que $43 \%$ (182) eran cognitivamente normales y $34 \%$ (143) tenían DCL, los demás presentaron demencia (23\%). Para definir el tamaño del efecto esperado se buscaron estudios similares, tomando los datos reportados por Petersen et al (24), en los que se evaluó una intervención con componentes cognitivos en pacientes con DCL, estimándose una tasa de conversión anual de DCL a demencia de $12 \%$, y una incidencia esperada de $4 \%$ de conversión anual de cognición normal a DCL $(24,25)$.

El tamaño de muestra se calculó considerando la fórmula de Perduzzi et al. 1996 (26).

Teniendo en cuenta que el tamaño de la población es conocida $(N)$ y una vez calculada la muestra $\left(n_{0}\right)$ se realizó el ajuste de corrección por población finita (fcp), mediante la fórmula de Araya et al. (27).

Considerando que se cuenta con una población de 182 sujetos con diagnóstico de cognición normal y con una incidencia esperada de 4\% de conversión anual de cognición normal a DCL se tomaron 146 sujetos normales

Para la población que pasa de DCL a demencia, con una población del estudio anterior de 143 sujetos con DCL y una incidencia esperada de $12 \%$ de conversión anual de DCL a demencia se calcularon 90 sujetos con DCL $(24,25)$. Así el tamaño de muestra total esperado fue de 236 sujetos.

Los sujetos fueron convocados telefónicamente a participar en el estudio y se invitaron a una primera reunión informativa del proyecto, donde además se dictó una conferencia de educación en salud y autocuidado de funciones intelectuales.

Los criterios de inclusión fueron tener edad mayor o igual a 50 años, ser autónomos, y haber sido diagnosticados como sujetos con cognición normal o DCL.

Se excluyeron sujetos con antecedente de enfermedad neuropsiquiátrica, déficit visual o auditivo severo y sujetos institucionalizados.

\section{Procedimiento de evaluación}

Los sujetos se citaban en grupos de 14 adultos con un familiar o acompañante para la evaluación de su función cognitiva en el Hospital Infantil Universitario de San José o en alguno de los salones comunales de las respectivas localidades, durante jornadas de mañana y tarde en los fines de semana, hasta completar la muestra requerida.

Previa firma de consentimiento informado se realizó la evaluación neuropsiquiátrica y neuropsicológica por el grupo de investigadores y estudiantes entrenados previamente, con el mismo protocolo del estudio anterior(12). Se documentaron sus riesgos cardiometabólicos y sociales, por autorreporte de antecedentes y se tomaron medidas antropométricas y la tensión arterial. Cada evaluación tenía una duración aproximada de tres horas.

El protocolo de evaluación incluyó lo siguiente: una historia y examen de neuropsiquiatría con un formulario de antecedentes de salud por autorreporte y aspectos sociodemográficos, el Minimental State Examination (MMSE) (28), la escala de Haschinski (29) y la figura del reloj (30). Se realizó la toma de la talla en centímetros con el sujeto descalzo, el peso en kilogramos (en una única balanza previamente calibrada) y la toma de la tensión arterial se realizó después de que el sujeto estuviera al menos cinco minutos sentado (con dos diferentes tensiómetros previamente calibrados). La evaluación psiquiátrica incluyó la evaluación del funcionamiento global con el índice de Barthel (ABVD) (31), escala de Lawton (AIVD) (32); comportamiento con la escala de Kertesz (33), depresión con la escala de depresión geriátrica de Yesavage (34), sobrecarga del cuidador con el test de Zarit (35), funcionalidad familiar con el índice de Apgar Familiar(36), y queja subjetiva de memoria del familiar (37).

La evaluación de neuropsicología incluía la siguiente batería: MoCA test validado para Colombia (38), TMT-A (39), prueba de memoria verbal de Grober y Buschke (40), queja subjetiva de memoria (QSM) (37), pruebas de fluidez verbal del lenguaje (41), test de denominación de 64 figuras (42), copia de la figura compleja de Rey (43), el test de inhibición de respuesta cognitiva Stroop (44), el test de digito - símbolo, refranes y semejanzas (45). 
Los residentes de medicina familiar y los estudiantes de psicología fueron entrenados previamente por el grupo de investigadores de clínica de memoria (neurólogos, psiquiatras y psicólogos) del Hospital Infantil Universitario de San José.

Los diagnósticos de demencia y deterioro cognitivo leve se definieron de acuerdo con los criterios del Manual Diagnóstico y Estadístico de los Trastornos Mentales (DSM-5) como trastorno neurocognitivo mayor y trastorno neurocognitivo menor. Estos diagnósticos se diferencian por la severidad de los síntomas (déficit de memoria, lenguaje, praxis, gnosias, funciones ejecutivas y cambios comportamentales o afectivos) determinados por la historia clínica y por el resultado de la pruebas realizadas, así como por su repercusión en la funcionalidad del paciente (ej: actividades de la vida diaria y actividades instrumentales) (46).

Una vez terminadas las evaluaciones, el grupo de investigadores procedía a analizar cada caso y establecía el diagnóstico por consenso, luego del cual se realizaba la entrega del resultado a cada participante junto a su acompañante, con una amplia explicación sobre la condición clínica encontrada y una remisión a su sistema de salud en los casos que progresaron a DCL o demencia. Al mismo tiempo se hacía entrega de una cartilla educativa, sobre factores de riesgo y prevención del deterioro cognitivo.

\section{Análisis Estadístico}

Las características sociodemográficas y los factores de riesgo cardiometabólico y sociales se describieron con frecuencias absolutas y relativas. Para describir la relación entre los factores de riesgo y la progresión del estado cognitivo, se realizó un análisis de correspondencias múltiples, tomando como variables ilustrativas la progresión de normal a DCL y de DCL a demencia, así como variables activas los factores de riesgo cardiometabólico y sociales. Se ajustó un modelo de regresión logística para la progresión de la población normal y DCL tomando como variables independientes los factores de riesgo considerados. El OR se calculó con un intervalo de confianza de $95 \%$. El análisis estadístico de la información se realizó en el programa STATA 13.

Aspectos éticos: el estudio fue aprobado por el Comité de Ética e Investigación con seres humanos del Hospital de San José y cumplió con los requisitos de la Declaración de Helsinki 2013 y de la Resolución 8430 de 1993 sobre ética e investigación en seres humanos en Colombia. El estudio se consideró con un nivel de riesgo mínimo.

\section{Resultados}

De un total de 236 pacientes localizados por teléfono se logró que asistieran a la valoración 215; desde 2014 fallecieron 13 pacientes y ocho declinaron asistir a la evaluación. Los 215 adultos autónomos mayores y funcionales de las diferentes localidades de Bogotá fueron evaluados entre octubre de 2017 y enero del 2018. Participaron 118 sujetos que tuvieron diagnóstico de cognición normal y 97 con diagnóstico de DCL en la evaluación anterior. Las características sociodemográficas y los factores de riesgo cardiovascular, metabólico y social se describen según el estado cognitivo encontrado en la Tabla 1. El 73\% de esta población fueron mujeres, con un promedio de edad de 71 (DS 7,3) años y de escolaridad de 8.2 (DE 5.4) años.

En un lapso de cuatro (DE1) años, un 3\% de los sujetos normales progresó a demencia, $22 \%$ a DCL, mientras que $75 \%$ permaneció estable. Por otra parte $10 \%$ de los sujetos con DCL progresó a demencia, $65 \%$ permaneció sin cambios y $25 \%$ regresó a un estado cognitivo normal (Tabla 1 ).

De los participantes que no cambiaron en el tiempo, estaban casados $53 \%$ de los sujetos normales y $48 \%$ de los sujetos con DCL. No se encontró que el estado civil tuviese un rol como factor de protección en estos sujetos ni en aquellos que tenían DCL y se normalizaron.

La mayoría de los sujetos en todos los grupos con o sin cambios cognitivos en el tiempo, no vivían solos.

En cuanto a la condición socioeconómica, en todos los grupos la mayoría de los sujetos estaban pensionados (aunque en la mayoría con salarios mínimos); no se encontró tampoco que esto fuera un factor de protección.

En la Figura 1 se representan los sujetos que tenían un estado cognitivo normal previo y las tres posibles evoluciones de su función cognitiva encontradas: progresión a demencia, progresión a DCL o permanecer normal, junto con los factores de riesgo relacionados con estas diferentes condiciones cognitivas. Los tres sujetos que estando previamente normales progresaron a demencia se caracterizaron por tener dos de estos sujetos niveles educativos altos (técnico y posgrado). El deterioro cognitivo tuvo un curso rápidamente progresivo en estos tres sujetos, uno presentaba una demencia frontotemporal, otra una degeneración corticobasal y la tercera una demencia tipo Alzheimer).

Quienes progresaron de un estado cognitivo normal a DCL se caracterizaron por tener baja escolaridad (primaria incompleta), un apgar familiar entre 13 y 16, dislipidemia y un rango de edad principalmente entre 70 y 79 años. Los sujetos sin cambio en el estado cognitivo se caracterizaron por ser en su mayoría mujeres con secundaria incompleta y tener un buen apgar familiar entre 17-20.

En la Figura 2 se representan los sujetos diagnosticados inicialmente como DCL y sus tres posibles evoluciones en el diagnóstico: progresión a demencia, recuperación a un estado cognitivo normal o permanecer sin cambio con DCL, junto con los factores de riesgo relacionados con esas evoluciones cognitivas. Los sujetos que progresaron a demencia se caracterizaron por tener baja escolaridad (primaria), o bachillerato incompleto, y depresión moderada a severa (yesavage de 6-9 o mayor de 10), mientras que los sujetos que pasaron de DCL a un estado de cognición normal, fueron en su mayoría mujeres casadas o divorciadas , con un buen apgar familiar y con educación técnica o universitaria.

Al realizar los análisis de regresión logística, encontramos que un bajo nivel educativo (considerado como $\leq 5$ años 
Tabla 1. Características sociodemográficas y factores de riesgo cardiometabólico y sociales según el estado cognitivo encontrados en los adultos mayores.

\begin{tabular}{|c|c|c|c|c|c|c|c|c|c|c|c|c|c|c|}
\hline \multirow{4}{*}{$\begin{array}{r}\text { Característica } \\
\text { Edad, Promedio (DE) }\end{array}$} & \multicolumn{6}{|c|}{ NORMAL (N=118) } & \multicolumn{6}{|c|}{ DETERIORO COGNITIVO LEVE(N=97) } & \multirow{2}{*}{\multicolumn{2}{|c|}{ Total }} \\
\hline & \multicolumn{2}{|c|}{ Igual a N } & \multicolumn{2}{|c|}{ N a DCL } & \multicolumn{2}{|c|}{ N a Demencia } & \multicolumn{2}{|c|}{ Igual a DCL } & \multicolumn{2}{|c|}{ DCL a N } & \multicolumn{2}{|c|}{ DCL a Demencia } & & \\
\hline & \multicolumn{2}{|c|}{$(n=89)(75 \%)$} & \multicolumn{2}{|c|}{$(n=26)(22 \%)$} & \multicolumn{2}{|c|}{$(\mathrm{n}=3)(3 \%)$} & \multicolumn{2}{|c|}{$(n=63)(65 \%)$} & \multicolumn{2}{|c|}{$(n=24)(25 \%)$} & \multicolumn{2}{|c|}{$(n=10)(10 \%)$} & \multicolumn{2}{|c|}{$(n=215)$} \\
\hline & 70.6 & (7.5) & 72.4 & $(8.1)$ & 68.6 & $(6.6)$ & 71.3 & $(7.6)$ & 70.8 & $(4.7)$ & 69.6 & (7.7) & 71 & $(7.3)$ \\
\hline \multicolumn{15}{|l|}{ Sexo } \\
\hline Femenino & 68 & $(76.4)$ & 22 & $(84.6)$ & 2 & $(66.7)$ & 44 & $(69.8)$ & 16 & $(66.6)$ & 4 & $(40)$ & 156 & $(72.5)$ \\
\hline Masculino & 21 & $(23.6)$ & 4 & $(15.4)$ & 1 & $(33.3)$ & 19 & $(30.2)$ & 8 & (33.3) & 6 & $(60)$ & 59 & $(27.5)$ \\
\hline \multicolumn{15}{|l|}{ Estado Civil } \\
\hline Casado & 47 & $(52.8)$ & 12 & $(46.1)$ & 1 & (33.3) & 30 & $(47.6)$ & 15 & $(62.5)$ & 4 & (40) & 109 & (50.7) \\
\hline Divorciado & 10 & (11.2) & 0 & $(0)$ & 1 & (33.3) & 4 & $(6.4)$ & 2 & $(8.3)$ & 3 & (30) & 20 & (9.3) \\
\hline Soltero & 11 & (12.4) & 4 & (15.4) & 1 & (33.3) & 10 & (15.9) & 3 & (12.5) & 0 & $(0)$ & 29 & (13.5) \\
\hline U. Libre & 5 & $(5.6)$ & 0 & (0) & 0 & (0) & 4 & $(6.3)$ & 1 & $(4.2)$ & 2 & (20) & 12 & (5.6) \\
\hline Viudo & 15 & (16.9) & 10 & $(38.5)$ & 0 & $(0)$ & 15 & (23.8) & 3 & $(12.5)$ & 1 & (10) & 44 & (20.5) \\
\hline Sin dato & 1 & (1.1) & 0 & (0) & 0 & (0) & 0 & (0) & 0 & (0) & 0 & (0) & 1 & $(0.4)$ \\
\hline \multicolumn{15}{|l|}{ Actividad económica } \\
\hline Activo & 20 & $(22.5)$ & 1 & (3.9) & 1 & (33.3) & 9 & (14.3) & 1 & $(4.2)$ & 1 & (10) & 33 & $(15.3)$ \\
\hline Dependiente & 16 & (18) & 5 & (19.2) & 0 & (0) & 14 & $(22.2)$ & 1 & $(4.2)$ & 3 & (30) & 39 & (18.1) \\
\hline Otros & 10 & (11.2) & 5 & (19.2) & 0 & (0) & 8 & (12.7) & 9 & $(37.5)$ & 2 & (20) & 34 & (15.9) \\
\hline Pensionado & 43 & $(48.3)$ & 15 & $(57.7)$ & 2 & $(66.4)$ & 32 & $(50.8)$ & 13 & $(54.1)$ & 4 & (40) & 109 & $(50.7)$ \\
\hline \multicolumn{15}{|l|}{ Vive solo } \\
\hline No & 78 & $(87.6)$ & 20 & (76.9) & 1 & $(33.3)$ & 56 & (88.9) & 20 & $(83.3)$ & 10 & (100) & 185 & $(86.1)$ \\
\hline $\mathrm{Si}$ & 11 & (12.4) & 6 & $(23.1)$ & 2 & $(66.7)$ & 7 & $(11.1)$ & 4 & $(16.7)$ & 0 & $(0)$ & 30 & (13.9) \\
\hline Sin dato & 0 & (0) & 0 & $(0)$ & 0 & (0) & 0 & (0) & 0 & (0) & 0 & (0) & 0 & (0) \\
\hline \multicolumn{15}{|l|}{ Escolaridad } \\
\hline $0-4$ & 18 & (20.3) & 10 & (38.5) & 0 & (0) & 20 & (31.8) & 6 & (25) & 2 & (20) & 56 & (26) \\
\hline 5 & 14 & (15.7) & 5 & (19.2) & 1 & $(33,3)$ & 17 & (27) & 2 & $(8.3)$ & 2 & (20) & 41 & (19.1) \\
\hline $6-10$ & 21 & (23.6) & 6 & $(23.1)$ & 0 & $(0)$ & 7 & (11.1) & 3 & (12.5) & 3 & (30) & 40 & (18.6) \\
\hline 11 & 9 & (10.1) & 0 & (0) & 0 & (0) & 6 & $(9.5)$ & 2 & $(8.3)$ & 0 & (0) & 17 & (8) \\
\hline $12-14$ & 9 & $(10.1)$ & 2 & $(7.7)$ & 0 & (0) & 4 & $(6.3)$ & 4 & $(16.7)$ & 0 & (0) & 19 & (8.8) \\
\hline $15-16$ & 4 & $(4.5)$ & 3 & (11.5) & 0 & (0) & 4 & $(6.3)$ & 5 & (20.9) & 3 & (30) & 19 & (8.8) \\
\hline$>17$ & 14 & (15.7) & 0 & (0) & 2 & $(66,7)$ & 5 & (8) & 2 & $(8.3)$ & 0 & $(0)$ & 23 & (10.7) \\
\hline Hipertensión & 40 & (44.9) & 9 & (34.6) & 0 & $(0)$ & 35 & (55.5) & 10 & (41.6) & 5 & (50) & 99 & (46) \\
\hline Diabetes & 15 & $(16.8)$ & 4 & (15.4) & 0 & (0) & 12 & (19) & 4 & (16.6) & 2 & (20) & 37 & $(17.2)$ \\
\hline Dislipidemia & 40 & (44.9) & 13 & $(50)$ & 0 & (0) & 31 & $(49.2)$ & 14 & (58.3) & 6 & (60) & 104 & (48.3) \\
\hline Obesidad & 12 & (13.4) & 4 & (15.4) & 0 & $(0)$ & 5 & (7.9) & 3 & (12.5) & 0 & $(0)$ & 24 & (11.1) \\
\hline
\end{tabular}


TRABAJOS ORIGINALES • Factores de riesgo modificables y deterioro cognitivo

Continuación... Tabla 1. Características sociodemográficas y factores de riesgo cardiometabólico y sociales según el estado cognitivo encontrados en los adultos mayores.

\begin{tabular}{|c|c|c|c|c|c|c|c|c|c|c|c|c|c|c|}
\hline \multirow{3}{*}{ Característica } & \multicolumn{6}{|c|}{ NORMAL $(\mathrm{N}=118)$} & \multicolumn{6}{|c|}{ DETERIORO COGNITIVO LEVE(N=97) } & \multirow{2}{*}{\multicolumn{2}{|c|}{ Total }} \\
\hline & \multicolumn{2}{|c|}{ Igual a N } & \multicolumn{2}{|c|}{ N a DCL } & \multicolumn{2}{|c|}{ N a Demencia } & \multicolumn{2}{|c|}{ Igual a DCL } & \multicolumn{2}{|c|}{ DCL a N } & \multicolumn{2}{|c|}{ DCL a Demencia } & & \\
\hline & \multicolumn{2}{|c|}{$(n=89)(75 \%)$} & \multicolumn{2}{|c|}{$(n=26)(22 \%)$} & \multicolumn{2}{|c|}{$(\mathrm{n}=3)(3 \%)$} & \multicolumn{2}{|c|}{$(n=63)(65 \%)$} & \multicolumn{2}{|c|}{$(n=24)(25 \%)$} & \multicolumn{2}{|c|}{$(n=10)(10 \%)$} & \multicolumn{2}{|c|}{$(n=215)$} \\
\hline \multicolumn{15}{|l|}{ Yesavage } \\
\hline $0-5$ & 75 & $(84.3)$ & 23 & $(88.5)$ & 3 & $(100)$ & 55 & $(87.3)$ & 21 & $(87.5)$ & 8 & $(80)$ & 185 & $(86)$ \\
\hline $6-9$ & 9 & $(10.1)$ & 3 & $(11.5)$ & 0 & (0) & 6 & $(9.5)$ & 3 & $(12.5)$ & 2 & $(20)$ & 23 & $(10.7)$ \\
\hline$\geq 10$ & 5 & $(5.6)$ & 0 & (0) & 0 & (0) & 2 & $(3.2)$ & 0 & $(0)$ & 0 & $(0)$ & 7 & (3.3) \\
\hline \multicolumn{15}{|l|}{ IMC } \\
\hline$\leq 25$ & 26 & $(29.2)$ & 12 & $(46.2)$ & 0 & (0) & 32 & $(50.8)$ & 9 & $(37.5)$ & 8 & (80) & 99 & $(46.0)$ \\
\hline$>25$ & 60 & (67.4) & 14 & (53.8) & 3 & (100) & 30 & (47.6) & 14 & $(58.3)$ & 2 & (20) & 111 & (51.6) \\
\hline Sin dato & 3 & ( 3.4$)$ & 0 & (0) & 0 & (0) & 1 & (1.6) & 1 & $(4.2)$ & 0 & (0) & 5 & (2.3) \\
\hline \multicolumn{15}{|l|}{ Edad } \\
\hline $50-59$ & 5 & $(5.7)$ & 1 & (3.9) & 1 & $(33,3)$ & 2 & $(3.2)$ & 0 & $(0)$ & 1 & (10) & 9 & $(4.2)$ \\
\hline $60-69$ & 41 & (46) & 9 & $(34.6)$ & 0 & (0) & 27 & $(42.8)$ & 11 & (45.8) & 5 & (50) & 94 & (43.7) \\
\hline $70-79$ & 29 & (32.6) & 10 & (38.5) & 2 & (66.7) & 23 & (36.5) & 13 & $(54.2)$ & 3 & (30) & 80 & (37.2) \\
\hline$\geq 80$ & 14 & (15.7) & 6 & (23) & 0 & (0) & 11 & (17.5) & 0 & (0) & 1 & (10) & 32 & (14.9) \\
\hline Depresión & 15 & (16.8) & 6 & (23) & 0 & (0) & 10 & (15.8) & 3 & (12.5) & 3 & (30) & 37 & (17.2) \\
\hline \multicolumn{15}{|l|}{ Apgar familiar } \\
\hline $17-20$ & 59 & $(66.2)$ & 15 & (57.6) & 1 & (33.3) & 42 & (66.6) & 15 & $(62.5)$ & 5 & (50) & 137 & (63.7) \\
\hline $13-16$ & 11 & (12.3) & 4 & (15.3) & 2 & (66.6) & 11 & (17.4) & 2 & (8.3) & 3 & (30) & 33 & (15.3) \\
\hline $10-12$ & 5 & (5.6) & 2 & (7.7) & 0 & (0) & 2 & $(3.1)$ & 0 & (0) & 1 & (10) & 10 & (4.6) \\
\hline$\leq 9$ & 5 & (5.6) & 2 & (7.7) & 0 & $(0)$ & 4 & (6.3) & 3 & (12.5) & 0 & (0) & 14 & $(6.5)$ \\
\hline Sin datos & 9 & (10.1) & 3 & (11.7) & 0 & (0) & 4 & $(6.3)$ & 4 & (16.6) & 1 & (10) & 21 & (9.8) \\
\hline
\end{tabular}

de escolaridad), se asoció con la progresión de un estado de cognición normal a DCL, con un OR=2.43 (IC95\%1.0045.91; $\mathrm{p}=0.049$ ); $\mathrm{y}$ en cuanto a los factores cardiometabólicos no se encontró asociación con el deterioro cognitivo, excepto que el tener un IMC $\leq 25$ se asoció con la progresión de DCL a demencia con un OR= 6.3 (IC95\% 1.26-31; $\mathrm{p}=0.025)$. De estos 10 sujetos con DCL previo que progresaron a demencia, siete experimentaron durante el tiempo de seguimiento una pérdida progresiva de peso, llevándolos a tener un IMC inferior o igual a 25; no se presentaron en ningún caso valores de IMC menores de 19, el rango estuvo de 20-25. Dos sujetos permanecieron sin cambios en su IMC, entre 23 y 24 y solo un sujeto aumento de peso cambiando su IMC de 24.4 a 26.1 .

En relación con la funcionalidad familiar (apgar familiar), se percibió en la mayoría de los casos estudiados como adecuada; sin embargo no se evidencio una asociación como factor protector.

\section{Discusión}

El objetivo del presente estudio, era evaluar si existía o no, una asociación entre los cambios cognitivos observados en el tiempo, en los sujetos que se habían encontrado normales o con DCL, en el estudio realizado entre 2012-2014, y los factores de riesgos cardiometabólicos o sociales.

Encontramos que tener una baja escolaridad ( $\leq 5$ años) se asoció al riesgo de progresión a DCL en los sujetos con cognición normal en un tiempo de $4( \pm 1)$ años. Esta asociación ya la habíamos descrito en el estudio transversal realizado entre 2012 y 2014, donde la baja escolaridad ( $\leq 5$ años) y el DCL presentaron un OR=1.96 (IC95\% 1.18-3.25); mientras que en demencia con escolaridades $\leq 4$ años o igual a 5 años, encontramos OR=11.20 (IC95\% 4.99-25.12) y OR= 7.91 (IC95\% (3.44-18.16) respectivamente (12).

En el estudio de Wei Xu. et, al (2015), los autores sugieren que la educación superior reduce significativamente el riesgo de demencia en forma lineal dosis-respuesta y que la 




Figura 1. Relación entre los factores de riesgo y los cambios cognitivos encontrados en los sujetos con diagnóstico inicial de cognición normal ( $N=118$ ). En el plano se presentan encerrados en los rectángulos los tres comportamientos diagnósticos y en los pequeños cuadros los factores de riesgo estudiados. Se sugiere una relación entre el comportamiento cognitivo encontrado, con el factor especialmente más cercano.

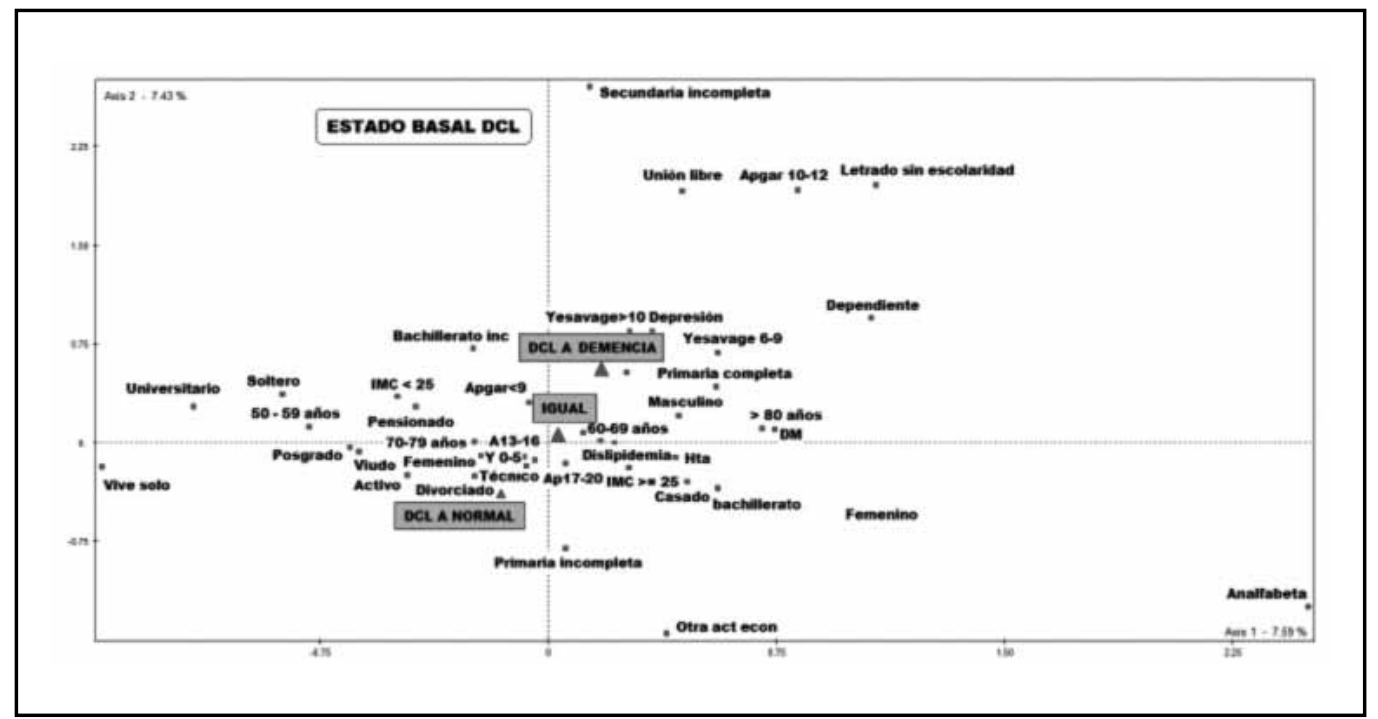

Figura 2. Relación entre los factores de riesgo y los cambios cognitivos encontrados en los sujetos con diagnóstico inicial de DCL (N=97). En el plano se presentan encerrados en los rectángulos los tres comportamientos diagnósticos y en los pequeños cuadros los factores de riesgo estudiados. Se sugiere una relación entre el comportamiento cognitivo encontrado, con el factor especialmente más cercano.

volumetría cerebral, el grosor cortical y el nivel de fosfoesfingolípidos, estarían asociados positivamente con la reserva cerebral y ayudarían a la transducción de señales cerebrales así como a la recuperación en la apoptosis cerebral. Otros autores sugieren que por cada año adicional de estudios se disminuiría en $7 \%$ el riesgo de demencia (10).

En nuestro estudio encontramos que $25 \%$ de los sujetos con DCL mejoraron su cognición revirtiendo a un estado normal, lo cual ya ha sido descrito en la literatura previamente. Larouche et al. (2015) describen como la psicoeducación (ej. usando métodos de mindfulness) controlaría factores de riesgo como la depresión, el estrés y el síndrome metabólico, mejorando en el cerebro los cambios por estrés oxidativo, neuroinflamación, así como los depósitos de beta amiloide y Tau, explicando de esta manera la recuperación o mejoras que se pueden observar en las funciones cognitivas de los individuos en el tiempo (47). En esta cohorte no realizamos un seguimiento del control de los factores de riesgo; sin embargo, esperamos analizarlos en un futuro estudio de seguimiento de esta misma cohorte. Por su parte Koepsell et al. 2012 en su estudio, describen 16\% de sujetos con DCL que revertían su déficit un año después de una evaluación inicial, encontrando que los sujetos con DCL unidominio no amnésicos, que no presentan el alelo APOE4, serían los de 
mayor posibilidad de recuperación. También se ha sugerido que el DCL sea una condición transitoria asociada a condiciones médicas o medicación subyacente (48).

La población de esta cohorte, había recibido una intervención con psicoeducación, cuando recibió al final del primer estudio el diagnóstico de su estado cognitivo, junto con las cartillas educativas que se les entregaron, sobre el manejo de los factores de riesgo para la protección del deterioro cognitivo.

En el caso de los tres sujetos que progresaron de un estado cognitivo normal a demencias rápidamente progresivas, no fue posible establecer asociaciones con factores de riesgo.

Los sujetos que teniendo un DCL previo progresaron a demencia, mostraron una asociación con un bajo índice de masa corporal $(\leq 25)$; en este sentido, existen estudios como el de Bell et al. (2017) que sugieren que la asociación entre el IMC bajo y un mayor riesgo de demencia puede ser el resultado indirecto de una fisiopatología temprana compartida con la enfermedad de Alzheimer debido al aumento de la neuroinflamación, la apoptosis y senescencia celular o podría representar una pérdida individual de la reserva fisiológica global. Estos autores proponen además que existiría una reducción de la incidencia de demencia de 3-5\% por cada aumento de la unidad, en el IMC en la edad tardía de la vida (22). De los 10 sujetos del estudio que progresaron de DCL a demencia, 7 presentaron una disminución del peso, que varió entre 3 y 10 kilos, durante el intervalo de tiempo entre las dos evaluaciones $(4 \pm 1$ años) llegando a valores de IMC que estaban en un rango entre 20 y 25 , que aunque normales, estaban más bajos que el que tenían en la primer evaluación. Esto sugiere que la pérdida de peso, aunque no llegue a valores de IMC menor de 19, estaría posiblemente relacionada con los mecanismos fisiopatológicos, en la mayoría de estos casos que progresaron a demencia. El amplio intervalo de confianza observado, se explica por el número reducido de sujetos que con DCL previo progreso a una demencia.

Aunque nuestro estudio no mostró una asociación significativa del deterioro cognitivo con factores cardiometabólicos, varios estudios hablan de dicha asociación. $\mathrm{Ng}$ et al. (2016) sugieren que tener síndrome metabólico, diabetes mellitus, obesidad central, dislipidemia o presentar tres o más factores de riesgo cardiovascular generaría $1.5-2$ veces más la posibilidad de desarrollar DCL (19).

Excepto la baja escolaridad que se asoció con la progresión de cognición normal a DCL, no se encontraron asociaciones entre los demás factores de riesgo social y el deterioro cognitivo. La población estudiada mostró en su mayoría buenas condiciones sociofamiliares.

El estudio de Sundström et al. (2018) propone que aquellos que viven solos (divorciados o solteros), pueden estar en riesgo de presentar demencia de inicio temprano y tardío, sugiriendo que las relaciones sociales deben considerarse como un factor de riesgo o protección para la demencia.
Una relación cercana puede ser una de las mejores fuentes de estimulación cognitiva y está asociada a una buena reserva cognitiva (49); por otra parte, una buena funcionalidad familiar ayudaría a tener una menor queja subjetiva de memoria como sugiere Kuiper et al. 2016 (23).

Entre las limitaciones del estudio está el tamaño de muestra para la conversión de sujetos con DCL a demencia: aunque se incluyeron más sujetos de los esperados (97 versus 90), la proporción de conversión fue menor a la calculada a una tasa de $12 \%$ anual, lo que para cuatro años daría un 39\%, se encontró en $10 \%$ en el presente estudio. Sin embargo, se encontró una conversión de $25 \%$ de los sujetos del grupo con DCL a una cognición normal. Por otra parte, el tiempo de seguimiento de $4( \pm 1)$ años, pudo ser corto, lo que impidió encontrar un mayor número de sujetos deteriorados, que permitiese a su vez evidenciar alguna relación con otros factores de riesgo. Esperamos a futuro, realizar una nueva evaluación de esta cohorte, en el contexto de nuevos estudios de seguimiento.

Las fortalezas del estudio las constituyen, el disponer de esta cohorte previamente estudiada y haberle realizado una nueva valoración con el mismo protocolo y por el mismo grupo de expertos.

\section{Conclusión}

En nuestra población tener baja escolaridad ( $\leq 5$ años) se asoció en los sujetos normales con un mayor riesgo de progresión a DCL, mientras que tener un IMC $\leq 25$ aumentaría el riesgo de progresión a demencia en los sujetos con DCL. No identificamos factores protectores en los sujetos que se normalizaron o que permanecieron sin cambios.

Requerimos de estudios más amplios, basados en cohortes de poblaciones comunitarias, de manera que podamos avanzar a corto plazo, en la identificación de los factores de riesgo, que permitan, al ser controlados, una reducción del riesgo de deterioro cognitivo en nuestra población. Un seguimiento a más largo plazo de la presente cohorte estudiada, está prevista.

\section{Agradecimientos}

A la población de adultos mayores y sus familias, a los estudiantes de psicología de la Universidad El Bosque, a la Fundación Universitaria de Ciencias de la Salud (FUCS) por la financiación del estudio.

\section{Referencias}

1. United Nations, Department of Economic and Social Affairs, Population Division (2015). World Population Ageing 2015.

2. Rizzi Liara R, Idiane Rosset. Global Epidemiology of Dementia: Alzheimer's and Vascular Types. BioMed Research International. 2014, : 8 pages

3. Mayeux R, Stern Y. Epidemiology of Alzheimer disease. Cold Spring Harb Perspect Med. 2012;2(8):2(8).

4. The Global Impact of Dementia. An Analysis of pevalence, Incidence, Cost and Trends.London,UK: . Alzheimer's Dissase International WAR. 2015.

5. Parra MA, Baez S, Allegri R, Nitrini R, Lopera F, Slachevsky A, et al. Dementia in Latin America: Assessing the present and envisioning the future. Neurology. 2018;90(5):222-31

6. César KG, Brucki SM, Takada LT, Nascimento LF, Gomes CM, Almeida MC, et al. Prevalence of Cognitive Impairment Without Dementia and Dementia in 
Tremembé, Brazil. Alzheimer Dis Assoc Disord. 2016;30(3):264-71.

7. Bowirrat A, Friedland RP, Korczyn AD. Vascular dementia among elderly Arabs in Wadi Ara. J Neurol Sci. 2002;203-204:73-6.

8. Ikejima C, Hisanaga A, Meguro K, Yamada T, Ouma S, Kawamuro Y, et al. Multicentre population-based dementia prevalence survey in Japan: a preliminary report. Psychogeriatrics. 2012;12(2):120-3.

9. Nguyen HT, Black SA, Ray LA, Espino DV, Markides KS. Predictors of decline in MMSE scores among older Mexican Americans. J Gerontol A Biol Sci Med Sci. 2002;57(3):M181-5.

10. Xu W, Tan L, Wang HF, Tan MS, Li JQ, Zhao QF, et al. Education and Risk of Dementia: Dose-Response Meta-Analysis of Prospective Cohort Studies. Mol Neurobiol. 2016;53(5):3113-23.

11.DANE_GEIH. Cálculos Ministerio de Salud y Protección Social. 2011.

12. Pedraza OL, Perilla HJ, Cruz A, Botero JA, Montalvo MC, Salazar AM, et al. Deterioro cognitivo y factores de riesgo cardiovascular y metabólico en una muestra de adultos de Bogotá. Acta Neurol Colomb. 2016;32:91-9.

13. Gooding MP, Amaya E, Parra M, Rios A M. Prevalencia de las demencias en el Municipio de Neiva 2003-2005. Acta Neurol Colomb. 2006; 22:243-8.

14. Díaz Cabezas Ricardo, Marulanda Mejía Felipe, Martínez Arias María Helena. Prevalencia de deterioro cognitivo y demencia en mayores de 65 años en una población urbana colombiana. Acta Neurol Colomb. [Internet]. 2013 July [cited 2018 Apr 21] ; 29( 3 ): 141-151

15. Pradilla A G, Vesga A BE, León-Sarmiento FE, GENECO. [National neuroepidemiological study in Colombia (EPINEURO)]. Rev Panam Salud Publica. 2003;14(2):104-11.

16. Pedraza OL, Salazar AM, Sierra FA, Montalvo MC, Muñoz Y, Díaz JM, et al. Mild cognitive impairment (MCI) and dementia in a sample of adults in the city of Bogotá. Dement Neuropsychol. 2017;11(3):262-9.

17. Lipnicki DM, Sachdev PS, Crawford J, Reppermund S, Kochan NA, Trollor JN, et al. Risk factors for late-life cognitive decline and variation with age and sex in the Sydney Memory and Ageing Study. PLoS One. 2013;8(6):e65841.

18. Alpérovitch A, Blachier M, Soumaré A, Ritchie K, Dartigues JF, RichardHarston S, et al. Blood pressure variability and risk of dementia in an elderly cohort, the Three-City Study. Alzheimers Dement. 2014;10(5 Suppl):S330-7.

19. Ng TP, Feng L, Nyunt MS, Gao Q, Lim ML, Collinson SL, et al. Metabolic Syndrome and the Risk of Mild Cognitive Impairment and Progression to Dementia: Follow-up of the Singapore Longitudinal Ageing Study Cohort. JAMA Neurol. 2016;73(4):456-63.

20. Baumgart M, Snyder HM, Carrillo MC, Fazio S, Kim H, Johns H. Summary of the evidence on modifiable risk factors for cognitive decline and dementia: A population-based perspective. Alzheimers Dement. 2015;11(6):718-26.

21. Qizilbash N, Gregson J, Johnson ME, Pearce N, Douglas I, Wing K, et al. $\mathrm{BMI}$ and risk of dementia in two million people over two decades: a retrospective cohort study. Lancet Diabetes Endocrinol. 2015;3(6):431-6.

22. Bell SP, Liu D, Samuels LR, Shah AS, Gifford KA, Hohman TJ, et al. Late-Life Body Mass Index, Rapid Weight Loss, Apolipoprotein E $\varepsilon 4$ and the Risk of Cognitive Decline and Incident Dementia. J Nutr Health Aging. 2017;21(10):1259-67.

23. Kuiper.S. The relationship between social functioning and subjective memory complaints in older persons: a population-based longitudinal cohort study. Int $J$ Geriatr Psychiatry 2016.

24. Petersen RC, Smith GE, Waring SC, Ivnik RJ, Tangalos EG, Kokmen E. Mild cognitive impairment: clinical characterization and outcome. Arch Neurol. 1999;56(3):303-8.

25. Barnes DE, Yaffe K. The projected effect of risk factor reduction on Alzheimer's disease prevalence. Lancet Neurol. 2011;10(9):819-28.

26. Peduzzi P , Concato J , Kemper E, Holford TR, Feinstein AR. A simulation study of the number of events per variable in logistic regression analysis. J Clin Epidemiol 1996 Dec; 49 (12): 1373-9.
27. Araya Alpízar C, Galindo Villardon MP. Tamaño de la muestra en investigación clínica. Medicina Clínica 20909; 133(1): 26-30.

28. Grigoletto F, Zappala G, Anderson D, Lebowitz B. Norms for the Mini-Mental State Examination in a healthy population. Neurology. 1999;53(2):315-20.

29. Hachinski VC, Lassen NA, Marshall J. Multi-infarct dementia. A cause of mental deterioration in the elderly. Lancet. 1974;2(7874):207-10.

30. Tuokko H, Hadjistavropoulos T, Miller JA, Beattie BL. The Clock Test: a sensitive measure to differentiate normal elderly from those with Alzheimer disease. $J$ Am Geriatr Soc. 1992; 40(6): 579-84.

31. Mahoney fi bd. Functional evaluation: the barthel index. Md State Med J 1965;14:61-5.

32. Lawton MP RD, Brody E, Kleban MH. The dynamics of caregiving for a demented elder among black and white families. J Gerontol. 1992;47(4):S156-64.

33. Kertesz A DW, Fox H. Frontal behavioral inventory: diagnostic criteria for frontal lobe dementia. Can J Neurol Sci 1997:24(1):9-36.

34. Yesavage JA BT, Rose TL, Lum O, Huang V, Adey M, et al. Development and validation of a geriatric depression screening scale: a preliminary report. $J$ Psychiatr Res. 1982:2;17(1):37-49.

35. Bédard M MD, Squire L, Dubois S, Lever JA, O'Donnell M. The Zari Burden Interview: a new short version and screening version. Gerontologist. 2001:41(5):652-7.

36. Smilkstein. The family APGAR: a proposal for a family function test and its use by physicians. Fam Pract 1978: 6:1231-9.

37. Cano C, Ruiz A, Plata S, Matallana D, Montañes P, Benito M, Et Al. Capacidad Operativa De Una Prueba De Tamizado En El Diagnóstico Temprano De La Enfermedad De Alzheimer [Operational Capacity Of A Screening Test In Early Diagnosis Of Alzheimer's Disease]. Revista De La Asociación Colombiana De Gerontología Y Geriatría 2002 16[3], 428-430.

38. Nasreddine ZS PN, Bédirian V, Charbonneau S, Whitehead V, Collin I, et al. The Montreal Cognitive Assessment, MoCA: a brief screening tool for mild cognitive impairment. J Am Geriatr Soc 2005;53(4):695-9.

39. Tombaugh. Trail Making Test A and B: normative data stratified by age and education. Arch Clin Neuropsychol 2004;19(2):203-14.

40. Grober E BH, Crystal H, Bang S, Dresner R. Screening for dementia by memory testing. Neurology 1988;38(6):900-3.

41. Tombaugh TN, Kozak J, Rees L. Normative data stratified by age and education for two measures of verbal fluency: FAS and animal naming. Arch Clin Neuropsychol. 1999;14(2):167-77.

42. Kaplan E, Goodglass H, Weintraub S. The Boston Naming Test. Experimental edition. Boston: Lippincott Williams and Wilkins. 1978.

43. Rey A. Test de copia de una figura compleja, Madrid, TEA. 1987.

44. Morrow. Normative data for the Stroop color word test for a North American population. Can J Neurol Sci 2013; 40(6): 842-7.

45. Wechsler D. Manual escala de Wechsler de inteligencia para adultos WAIS III México. Editorial: Manual Moderno. 2003

46. Sachdev PS, Blacker D, Blazer DG, Ganguli M, Jeste DV, Paulsen JS, et al. Classifying neurocognitive disorders: the DSM-5 approach. Nat Rev Neurol. 2014;10(11):634-42.

47. Larouche E, Hudon C, Goulet S. Potential benefits of mindfulness-based interventions in mild cognitive impairment and Alzheimer's disease: an interdisciplinary perspective. Behav Brain Res. 2015;276:199-212.

48. Koepsell TD, Monsell SE. Reversion from mild cognitive impairment to normal or near-normal cognition: risk factors and prognosis. Neurology. 2012;79(15):1591-8.

49. Sundström A, Westerlund O, Kotyrlo E. Marital status and risk of dementia: a nationwide population-based prospective study from Sweden. BMJ Open. 2016;6(1):e008565. 